\title{
COBALT FERRITE FILMS AS DESCRIBED BY THIRD ORDER PERTURBED
} HEISENBERG HAMILTONIAN

\section{P. SAMARASEKARA*}

\section{Department of Physics, University of Peradeniya, Peradeniya, Sri Lanka.}

\begin{abstract}
Magnetic properties of thick films of Cobalt ferrite have been investigated using third order perturbed modified Heisenberg Hamiltonian at different values of second and fourth order magnetic anisotropy. Thick films with up to 10,000 layers have been employed for this investigation. The magnetic energy versus number of layers, second and fourth order anisotropy have been plotted in order to find the magnetically easy and hard directions of cobalt ferrite films. Energy has minimum and maximum values at certain values of second and fourth order anisotropy values, indicating that that there are magnetically easy and hard directions. Energy shows the same type of variations with number of layers in the film. For the values considered in this manuscript, the easy direction of magnetization was found to be $153^{0}$ with the normal line drawn to the film surface. However, the easy axis direction depends on the values of parameters used in simulation. Determination of easy directions of magnetic films is important in applications of magnetic memory devices.
\end{abstract}

Keywords: Cobalt ferrite, thick films, Heisenberg Hamiltonian, perturbation

\section{INTRODUCTION}

Cobalt ferrite finds potential applications in, magnetic memory devices, microwave devices, hydrogen production devices (Rajendra et al., 2011), microelectromechanical system devices, stress sensors, actuators and magnetic nanobowls. Cobalt ferrite magnetic thin films have been synthesized using electrostatic

\footnotetext{
*Corresponding author Email: pubudus@pdn.ac.lk
} 
spray method (Rajendra et al., 2011), pulsed laser deposition (Raghunathan et al., 2010, Thang et al., 2007, Araujo et al., 2008), electrophoresis (Barbosa et al., 2010). But theoretical investigations of cobalt ferrite films haven't been conducted using any theoretical model. The thin and thick films of Nickel ferrite have been theoretically investigated using non-perturbed (Samarasekara, 2007), $2^{\text {nd }}$ order (Samarasekara et al., 2009, Samarasekara, 2010) and $3^{\text {rd }}$ order (Samarasekara \& Mendoza, 2011, Samarasekara, 2011) perturbed Heisenberg Hamiltonian by us previously. The variation of magnetic energy with number of layers \& stress induced anisotropy were plotted and the easy \& hard directions were found using non-perturbed modified Heisenberg Hamiltonian (Samarasekara, 2007). The magnetic energy versus angle of spins \& stress induced anisotropy were plotted and the easy $\&$ hard directions were calculated using the $2^{\text {nd }}$ order perturbed modified Heisenberg Hamiltonian (Samarasekara et al., 2009, Samarasekara, 2010). In addition, the variation of magnetic energy with number of layers $\&$ stress induced anisotropy were plotted and the easy \& hard directions were found using the $3^{\text {rd }}$ order perturbed modified Heisenberg Hamiltonian (Samarasekara \& Mendoza, 2011, Samarasekara, 2011).

In this manuscript, the magnetic energy versus second order and fourth order magnetic anisotropy was plotted to find the easy and hard directions of magnetization using the $3^{\text {rd }}$ order perturbed Heisenberg Hamiltonian. MATLAB was used to prepare all the graphs. The inverse spinel structure of $\mathrm{Fe}^{3+}\left(\mathrm{Co}^{2+} \mathrm{Fe}^{3+}\right) \mathrm{O}_{4}$ was investigated. The spins of $\mathrm{Co}^{2+}$ and $\mathrm{Fe}^{3+}$ were considered to be $3 \mu_{\mathrm{B}}$ and $5 \mu_{\mathrm{B}}$, respectively. An equation for the magnetic energy per unit spin of Cobalt was derived using $3^{\text {rd }}$ order perturbed Heisenberg Hamiltonian.

\section{MODEL}

Following modified Hamiltonian was used as the model.

$$
\begin{aligned}
H= & -J \sum_{m, n} \vec{S}_{m} \vec{S}_{n}+\omega \sum_{m \neq n}\left(\frac{\vec{S}_{m} \cdot \vec{S}_{n}}{r_{m n}{ }^{3}}-\frac{3\left(\vec{S}_{m} \cdot \vec{r}_{m n}\right)\left(\vec{r}_{m n} \cdot \vec{S}_{n}\right)}{r_{m n}{ }^{5}}\right)-\sum_{m} D_{\lambda_{m}}{ }^{(2)}\left(S_{m}{ }^{z}\right)^{2}-\sum_{m} D_{\lambda_{m}}{ }^{(4)}\left(S_{m}{ }^{z}\right)^{4} \\
& -\sum_{m} \vec{H} \cdot \vec{S}_{m}-\sum_{m} K_{s} \operatorname{Sin} 2 \theta_{m}
\end{aligned}
$$

Here, first, second, third and fourth terms represent the spin exchange interaction, magnetic dipole interaction, second order anisotropy and fourth order anisotropy, respectively. The indices of planes are denoted by $\mathrm{m}$ and $\mathrm{n}$, and they vary from 1 to $\mathrm{N}$ for 
a film with N number of layers. $\vec{S}_{i}$ is a spin vector with unit length at point $\vec{r}_{i}$ in layer $\lambda_{i}$. Therefore the ground state energy will be calculated per spin with Z-axis normal to film plane. $\vec{H}$ is the external magnetic field with the effective magnetic moment of the spins incorporated. $\omega=\frac{\mu_{0} \mu^{2}}{4 \pi a^{3}}$ is the strength of long range dipole interaction on a lattice with lattice constant a, where $\mu_{0}$ is the permeability. $r_{m n}$ is the separation between two spins $s_{m}$ and $\mathrm{s} . D_{\lambda_{m}}{ }^{(2)}$ and $D_{\lambda_{m}}{ }^{(4)}$ are second and fourth order magnetic anisotropy constants of the lattice, respectively. Here, the last term indicates the change of magnetic energy under the influence of a stress. $\mathrm{K}_{\mathrm{s}}$ depends on the product of magnetostriction coefficient $\left(\lambda_{s}\right)$ and the stress $(\sigma)$. Here $\theta$ is the angle between local magnetization (M) and the stress. Within a single domain, $\mathrm{M}$ is parallel to the spin. If stress is applied normal to the film plane, then $\theta_{\mathrm{m}}$ is the angle between a perpendicular line drawn to the film plane and the local spin. Here $\mathrm{K}_{\mathrm{s}}$ can be positive or negative depending on the type of stress whether it is compressive or tensile.

The cubic cell has been divided into 8 spin layers with alternative $\mathrm{A}$ and $\mathrm{Fe}$ spins layers. The spins of A and Fe will be taken as 1 and p, respectively. While the spins in one layer point in one direction, spins in adjacent layers point in opposite directions. A thin film with (001) spinel cubic cell orientation will be considered. The length of one side of unit cell will be taken as "a". Within the cell the spins orient in one direction due to the super exchange interaction between spins (or magnetic moments). Therefore the results proven for oriented case in one of our early reports will be used for following equations (Samarasekara, 2007) but the angle $\theta$ will vary from $\theta_{\mathrm{m}}$ to $\theta_{\mathrm{m}+1}$ at the interface between two cells.

For a thin film with thickness $\mathrm{Na}$,

Spin interaction energy $=\mathrm{E}_{\text {exchange }}=\mathrm{N}\left(-10 \mathrm{~J}+72 \mathrm{Jp}-22 \mathrm{Jp} \mathrm{p}^{2}\right)+8 \mathrm{Jp} \sum_{m=1}^{N-1} \cos \left(\theta_{m+1}-\theta_{m}\right)$

Dipole interaction energy $=\mathrm{E}_{\text {dipole }}$

$$
E_{\text {dipole }}=-48.415 \omega \sum_{m=1}^{N}\left(1+3 \cos 2 \theta_{m}\right)+20.41 \omega p \sum_{m=1}^{N-1}\left[\cos \left(\theta_{m+1}-\theta_{m}\right)+3 \cos \left(\theta_{m+1}+\theta_{m}\right)\right]
$$

Here the first and second term in each above equation represent the variation of energy within the cell and the interface of the cell, respectively. Then total energy is given by 


$$
\begin{aligned}
\mathrm{E}(\theta) & =\mathrm{N}\left(-10 \mathrm{~J}+72 \mathrm{Jp}-22 \mathrm{Jp} \mathrm{p}^{2}\right)+8 \mathrm{Jp} \sum_{m=1}^{N-1} \cos \left(\theta_{m+1}-\theta_{m}\right) \\
& -48.415 \omega \sum_{m=1}^{N}\left(1+3 \cos 2 \theta_{m}\right)+20.41 \omega p \sum_{m=1}^{N-1}\left[\cos \left(\theta_{m+1}-\theta_{m}\right)+3 \cos \left(\theta_{m+1}+\theta_{m}\right)\right] \\
& -\sum_{m=1}^{N}\left[D_{m}{ }^{(2)} \cos ^{2} \theta_{m}+D_{m}{ }^{(4)} \cos ^{4} \theta_{m}\right] \\
& -4(1-p) \sum_{m=1}^{N}\left[H_{\text {in }} \sin \theta_{m}+H_{\text {out }} \cos \theta_{m}+K_{s} \sin 2 \theta_{m}\right]
\end{aligned}
$$

Here the anisotropy energy term and the last term have been explained in our previous report for oriented spinel ferrite (Samarasekara, 2007). If the angle is given by $\theta_{\mathrm{m}}=\theta+\varepsilon_{\mathrm{m}}$ with perturbation $\varepsilon_{\mathrm{m}}$, after taking the terms up to third order perturbation of $\varepsilon$, The total energy can be given as $\mathrm{E}(\theta)=\mathrm{E}_{0}+\mathrm{E}(\varepsilon)+\mathrm{E}\left(\varepsilon^{2}\right)+\mathrm{E}\left(\varepsilon^{3}\right)$

Here

$$
\begin{aligned}
& \mathrm{E}_{0}=-10 \mathrm{JN}+72 \mathrm{pNJ}-22 \mathrm{~J} \mathrm{p}^{2} \mathrm{~N}+8 \mathrm{~J} \mathrm{p}(\mathrm{N}-1)-48.415 \omega \mathrm{N}-145.245 \omega \mathrm{N} \cos (2 \theta) \\
& +20.41 \omega \mathrm{p}[(\mathrm{N}-1)+3(\mathrm{~N}-1) \cos (2 \theta)] \\
& -\cos ^{2} \theta \sum_{m=1}^{N} D_{m}{ }^{(2)}-\cos ^{4} \theta \sum_{m=1}^{N} D_{m}{ }^{(4)}-4(1-p) N\left(H_{\text {in }} \sin \theta+H_{\text {out }} \cos \theta+K_{s} \sin 2 \theta\right) \\
& E(\varepsilon)=290.5 \omega \sin (2 \theta) \sum_{m=1}^{N} \varepsilon_{m}-61.23 \omega p \sin (2 \theta) \sum_{m=1}^{N-1}\left(\varepsilon_{m}+\varepsilon_{n}\right) \\
& +\sin 2 \theta \sum_{m=1}^{N} D_{m}^{(2)} \varepsilon_{m}+2 \cos ^{2} \theta \sin 2 \theta \sum_{m=1}^{N} D_{m}^{(4)} \varepsilon_{m} \\
& +4(1-p)\left[-H_{\text {in }} \cos \theta \sum_{m=1}^{N} \varepsilon_{m}+H_{\text {out }} \sin \theta \sum_{m=1}^{N} \varepsilon_{m}-2 K_{s} \cos 2 \theta \sum_{m=1}^{N} \varepsilon_{m}\right] \\
& E\left(\varepsilon^{2}\right)=-4 J p \sum_{m=1}^{N-1}\left(\varepsilon_{n}-\varepsilon_{m}\right)^{2}+290.5 \omega \cos (2 \theta) \sum_{m=1}^{N}{\varepsilon_{m}}^{2}-10.2 \omega p \sum_{m=1}^{N-1}\left(\varepsilon_{n}-\varepsilon_{m}\right)^{2} \\
& -30.6 \omega p \cos (2 \theta) \sum_{m=1}^{N-1}\left(\varepsilon_{n}+\varepsilon_{m}\right)^{2} \\
& +\cos 2 \theta \sum_{m=1}^{N} D_{m}{ }^{(2)} \varepsilon_{m}{ }^{2}+2 \cos ^{2} \theta\left(\cos ^{2} \theta-3 \sin ^{2} \theta\right) \sum_{m=1}^{N} D_{m}{ }^{(4)} \varepsilon_{m}{ }^{2} \\
& +4(1-p)\left[\frac{H_{\text {in }}}{2} \sin \theta \sum_{m=1}^{N}{\varepsilon_{m}}^{2}+\frac{H_{\text {out }}}{2} \cos \theta \sum_{m=1}^{N}{\varepsilon_{m}}^{2}+2 K_{s} \sin 2 \theta \sum_{m=1}^{N} \varepsilon_{m}{ }^{2}\right]
\end{aligned}
$$




$$
\begin{aligned}
E\left(\varepsilon^{3}\right)= & 10.2 p \omega \sin 2 \theta \sum_{m, n=1}^{N}\left(\varepsilon_{m}+\varepsilon_{n}\right)^{3}-193.66 \omega \sin 2 \theta \sum_{m=1}^{N} \varepsilon_{m}{ }^{3}-\frac{4}{3} \cos \theta \sin \theta \sum_{m=1}^{N} D_{m}{ }^{(2)} \varepsilon_{m}{ }^{3} \\
& -4 \cos \theta \sin \theta\left(\frac{5}{3} \cos ^{2} \theta-\sin ^{2} \theta\right) \sum_{m=1}^{N} D_{m}{ }^{(4)} \varepsilon_{m}{ }^{3} \\
& +4(1-p)\left[\frac{H_{\text {in }}}{6} \cos \theta \sum_{m=1}^{N} \varepsilon_{m}{ }^{3}-\frac{H_{\text {out }}}{6} \sin \theta \sum_{m=1}^{N} \varepsilon_{m}{ }^{3}+\frac{4 K_{s}}{3} \cos 2 \theta \sum_{m=1}^{N} \varepsilon_{m}{ }^{3}\right]
\end{aligned}
$$

The sin and cosine terms in equation number 2 have been expanded to obtain above equations. Here $\mathrm{n}=\mathrm{m}+1$.

Under the constraint $\sum_{m=1}^{N} \varepsilon_{m}=0$, first and last three terms of equation 4 are zero.

Therefore, $\mathrm{E}(\varepsilon)=\vec{\alpha} \cdot \vec{\varepsilon}$

Here $\vec{\alpha}(\varepsilon)=\vec{B}(\theta) \sin 2 \theta$ are the terms of matrices with

$$
B_{\lambda}(\theta)=-122.46 \omega p+D_{\lambda}^{(2)}+2 D_{\lambda}^{(4)} \cos ^{2} \theta
$$

Also $E\left(\varepsilon^{2}\right)=\frac{1}{2} \vec{\varepsilon} \cdot C \cdot \vec{\varepsilon}$, and matrix $\mathrm{C}$ is assumed to be symmetric $\left(\mathrm{C}_{\mathrm{mn}}=\mathrm{C}_{\mathrm{nm}}\right)$.

Here the elements of matrix $\mathrm{C}$ can be given as following,

$\mathrm{C}_{\mathrm{m}, \mathrm{m}+1}=8 \mathrm{Jp}+20.4 \omega \mathrm{p}-61.2 \mathrm{p} \omega \cos (2 \theta)$

For $\mathrm{m}=1$ and $\mathrm{N}$,

$$
\begin{aligned}
\mathrm{C}_{\mathrm{mm}} & =-8 \mathrm{Jp}-20.4 \omega \mathrm{p}-61.2 \mathrm{p} \omega \cos (2 \theta)+581 \omega \cos (2 \theta)+2 \cos 2 \theta D_{m}{ }^{(2)} \\
& +4 \cos ^{2} \theta\left(\cos ^{2} \theta-3 \sin ^{2} \theta\right) D_{m}{ }^{(4)}+4(1-p)\left[H_{\text {in }} \sin \theta+H_{\text {out }} \cos \theta+4 K_{s} \sin (2 \theta)\right]
\end{aligned}
$$

For $\mathrm{m}=2,3,----, \mathrm{N}-1$

$$
\begin{aligned}
\mathrm{C}_{\mathrm{mm}} & =-16 \mathrm{Jp}-40.8 \omega \mathrm{p}-122.4 \mathrm{p} \omega \cos (2 \theta)+581 \omega \cos (2 \theta)+2 \cos 2 \theta D_{m}{ }^{(2)} \\
& +4 \cos ^{2} \theta\left(\cos ^{2} \theta-3 \sin ^{2} \theta\right) D_{m}{ }^{(4)}+4(1-p)\left[H_{\text {in }} \sin \theta+H_{\text {out }} \cos \theta+4 K_{s} \sin (2 \theta)\right]
\end{aligned}
$$

Otherwise, $\mathrm{C}_{\mathrm{mn}}=0$

Also $E\left(\varepsilon^{3}\right)=\varepsilon^{2} \beta . \vec{\varepsilon}$ 
Here matrix elements of matrix $\beta$ can be given as following.

When $\mathrm{m}=1$ and $\mathrm{N}$,

$$
\begin{aligned}
\beta_{m m} & =-193.66 \omega \sin 2 \theta+10.2 p \omega \sin 2 \theta-\frac{4}{3} \cos \theta \sin \theta D_{m}{ }^{(2)} \\
& -4 \cos \theta \sin \theta\left(\frac{5}{3} \cos ^{2} \theta-\sin ^{2} \theta\right) D_{m}{ }^{(4)}+4(1-p)\left[\frac{H_{\text {in }}}{6} \cos \theta-\frac{H_{\text {out }}}{6} \sin \theta+\frac{4 K_{s}}{3} \cos 2 \theta\right]
\end{aligned}
$$

When $\mathrm{m}=2,3,------, \mathrm{N}-1$

$$
\begin{aligned}
& \beta_{m m}=-193.66 \omega \sin 2 \theta+20.4 p \omega \sin 2 \theta-\frac{4}{3} \cos \theta \sin \theta D_{m}{ }^{(2)} \\
& -4 \cos \theta \sin \theta\left(\frac{5}{3} \cos ^{2} \theta-\sin ^{2} \theta\right) D_{m}{ }^{(4)}+4(1-p)\left[\frac{H_{\text {in }}}{6} \cos \theta-\frac{H_{\text {out }}}{6} \sin \theta+\frac{4 K_{s}}{3} \cos 2 \theta\right] \\
& \beta_{m, m+1}=30.6 p \omega \sin 2 \theta
\end{aligned}
$$

Otherwise $\beta_{\mathrm{nm}}=0$. Also matrix $\beta$ is symmetric such that $\beta_{\mathrm{nm}}=\beta_{\mathrm{mn}}$.

Finally, the total magnetic energy given in equation 2 can be deduced to

$$
\mathrm{E}(\theta)=\mathrm{E}_{0}+\vec{\alpha} \cdot \vec{\varepsilon}+\frac{1}{2} \vec{\varepsilon} \cdot C \cdot \vec{\varepsilon}+\varepsilon^{2} \beta \cdot \vec{\varepsilon}
$$

Only the second order terms of $\varepsilon$ will be considered for following derivation, since the derivation with the third order terms of $\varepsilon$ in above equation is tedious.

Then $\mathrm{E}(\theta)=\mathrm{E}_{0}+\vec{\alpha} \cdot \vec{\varepsilon}+\frac{1}{2} \vec{\varepsilon} \cdot C \cdot \vec{\varepsilon}$

Using a suitable constraint in above equation, it is possible to show that $\vec{\varepsilon}=-C^{+} \cdot \vec{\alpha}$ Here $\mathrm{C}^{+}$is the pseudo-inverse given by

$$
\text { C. } C^{+}=1-\frac{E}{N} \text {. }
$$

Each element in matrix $E$ is 1 .

After using $\vec{\varepsilon}$ in equation $9, \mathrm{E}(\theta)=\mathrm{E}_{0}-\frac{1}{2} \vec{\alpha} \cdot C^{+} \cdot \vec{\alpha}-\left(C^{+} \alpha\right)^{2} \vec{\beta}\left(C^{+} \alpha\right)$

\section{RESULTS AND DISCUSSION}

Value of $\mathrm{p}$ for cobalt ferrite is 1.67 . According to equation $10, \mathrm{C}^{+}$is the standard inverse matrix of $\mathrm{C}$, when $\mathrm{N}$ is really large. When the difference between $\mathrm{m}$ and $\mathrm{n}$ is one, $\mathrm{C}_{\mathrm{m}, \mathrm{m}+1}=8 \mathrm{Jp}+20.4 \omega \mathrm{p}-61.2 \mathrm{p} \omega \cos (2 \theta)$. If $\mathrm{H}_{\mathrm{in}}, \mathrm{H}_{\text {out }}$ and $\mathrm{K}_{\mathrm{s}}$ are very large, then $\mathrm{C}_{11}>\mathrm{C}_{12}$. 
If this $\mathrm{C}_{\mathrm{m}, \mathrm{m}+1}=0$, then the matrix $\mathrm{C}$ becomes diagonal, and the elements of inverse matrix $\mathrm{C}^{+}$is given by $C^{+}{ }_{m m}=\frac{1}{C_{m m}}$. Therefore all the derivation will be done under above assumption to avoid tedious derivations.

From equation 3 ,

$\mathrm{E}_{0}=-10 \mathrm{JN}+120.24 \mathrm{NJ}-61.36 \mathrm{JN}+13.36 \mathrm{~J}(\mathrm{~N}-1)-48.415 \omega \mathrm{N}-145.245 \omega \mathrm{N} \cos (2 \theta)$

$+34.1 \omega[(\mathrm{N}-1)+3(\mathrm{~N}-1) \cos (2 \theta)]$

From equation 7 ,

$\mathrm{C}_{11}=\mathrm{C}_{\mathrm{NN}}=-13.36 \mathrm{~J}-34.07 \omega+478.8 \omega \cos (2 \theta)+2 \cos 2 \theta D_{m}{ }^{(2)}$

$+4 \cos ^{2} \theta\left(\cos ^{2} \theta-3 \sin ^{2} \theta\right) D_{m}{ }^{(4)}-2.68\left[H_{\text {in }} \sin \theta+H_{\text {out }} \cos \theta+4 K_{s} \sin (2 \theta)\right]$

For $\mathrm{m}=2,3,----, \mathrm{N}-1$

$\mathrm{C}_{22}=\mathrm{C}_{33}=----=\mathrm{C}_{\mathrm{N}-1, \mathrm{~N}-1}=-26.72 \mathrm{~J}-68.14 \omega+376.59 \omega \cos (2 \theta)+2 \cos 2 \theta D_{m}{ }^{(2)}$

$+4 \cos ^{2} \theta\left(\cos ^{2} \theta-3 \sin ^{2} \theta\right) D_{m}^{(4)}-2.68\left[H_{\text {in }} \sin \theta+H_{\text {out }} \cos \theta+4 K_{s} \sin (2 \theta)\right]$

From equation 6 ,

$$
\begin{aligned}
& \alpha_{1}=\left[-306.15 \omega+D_{\lambda}{ }^{(2)}+2 D_{\lambda}{ }^{(4)} \cos ^{2} \theta\right] \sin (2 \theta) \\
& \beta_{11}=\beta_{N N}=-168.16 \omega \sin 2 \theta-\frac{4}{3} \cos \theta \sin \theta D_{m}{ }^{(2)} \\
& -4 \cos \theta \sin \theta\left(\frac{5}{3} \cos ^{2} \theta-\sin ^{2} \theta\right) D_{m}{ }^{(4)}-6\left[\frac{H_{\text {in }}}{6} \cos \theta-\frac{H_{\text {out }}}{6} \sin \theta+\frac{4 K_{s}}{3} \cos 2 \theta\right] \\
& \beta_{22}=-142.66 \omega \sin 2 \theta-\frac{4}{3} \cos \theta \sin \theta D_{m}{ }^{(2)} \\
& -4 \cos \theta \sin \theta\left(\frac{5}{3} \cos ^{2} \theta-\sin ^{2} \theta\right) D_{m}{ }^{(4)}-6\left[\frac{H_{\text {in }}}{6} \cos \theta-\frac{H_{\text {out }}}{6} \sin \theta+\frac{4 K_{s}}{3} \cos 2 \theta\right] \\
& \beta_{m, m+1}=76.5 \omega \sin 2 \theta \\
& \left(\mathrm{C}^{+} \alpha\right)^{2} \beta\left(\mathrm{C}^{+} \alpha\right)=\left(\mathrm{C}_{11}{ }^{+} \alpha_{1}\right)^{2}\left(\beta_{11} \mathrm{C}_{11}{ }^{+} \alpha_{1}+\beta_{12} \mathrm{C}_{22}{ }^{+} \alpha_{2}+-------+\beta_{1 \mathrm{~N}} \mathrm{C}_{\mathrm{NN}}{ }^{+} \alpha_{\mathrm{N}}\right) \\
& +\left(\mathrm{C}_{22}{ }^{+} \alpha_{2}\right)^{2}\left(\beta_{21} \mathrm{C}_{11}{ }^{+} \alpha_{1}+\beta_{22} \mathrm{C}_{22}{ }^{+} \alpha_{2}+-------+\beta_{2 \mathrm{~N}} \mathrm{C}_{\mathrm{NN}}{ }^{+} \alpha_{\mathrm{N}}\right) \\
& +\left(\mathrm{C}_{33}{ }^{+} \alpha_{3}\right)^{2}\left(\beta_{31} \mathrm{C}_{11}{ }^{+} \alpha_{1}+\beta_{32} \mathrm{C}_{22}{ }^{+} \alpha_{2}+-------+\beta_{3 \mathrm{~N}} \mathrm{C}_{\mathrm{NN}}{ }^{+} \alpha_{\mathrm{N}}\right)+-------- \\
& \text {------ }+\left(\mathrm{C}_{\mathrm{NN}}^{+} \alpha_{\mathrm{N}}\right)^{2}\left(\beta_{\mathrm{N} 1} \mathrm{C}_{11}{ }^{+} \alpha_{1}+\beta_{\mathrm{N} 2} \mathrm{C}_{22}{ }^{+} \alpha_{2}+-------+\beta_{\mathrm{NN}} \mathrm{C}_{\mathrm{NN}}{ }^{+} \alpha_{\mathrm{N}}\right)
\end{aligned}
$$

$\left(C^{+} \alpha\right)^{2} \beta\left(C^{+} \alpha\right)=\alpha^{3}\left[\frac{2}{C_{11}{ }^{2}}\left(\frac{\beta_{11}}{C_{11}}+\frac{\beta_{12}}{C_{22}}\right)+\frac{2}{C_{22}{ }^{2}}\left(\frac{\beta_{12}}{C_{11}}+\frac{\beta_{22}+\beta_{12}}{C_{22}}\right)+\frac{N-4}{C_{22}{ }^{3}}\left(2 \beta_{12}+\beta_{22}\right)\right]$ 
All the simulations given in Figure 1 and 2 have been performed for films with 10000 layers $(\mathrm{N}=10,000)$. The variation of total magnetic energy given in equation 11 was plotted against angle and $\frac{D_{m}{ }^{(4)}}{\omega}$ as shown in figure 1 . The other values were kept at $\frac{J}{\omega}=\frac{D_{m}{ }^{(2)}}{\omega}=\frac{H_{\text {in }}}{\omega}=\frac{H_{\text {out }}}{\omega}=\frac{K_{s}}{\omega}=10$ for this simulation. Because the ratios between energies have been considered, all the parameters on axes are dimensionless. According to this 3-D plot, energy is a minimum at certain values of $\frac{D_{m}{ }^{(4)}}{\omega}$ such as $\frac{D_{m}{ }^{(4)}}{\omega}=27,38,41,52,55,58---$ etc. This implies that the film can be easily oriented along the easy direction for $\frac{D_{m}{ }^{(4)}}{\omega}=27,38,41,52,55,58---$ etc. Also the energy is a maximum at $\frac{D_{m}{ }^{(4)}}{\omega}=21,33,39,47,---$ etc, implying that it is difficult to orient the film along the hard direction at these values of $\frac{D_{m}{ }^{(4)}}{\omega}$. There are several certain easy and hard directions at any of these $\frac{D_{m}^{(4)}}{\omega}$ values.

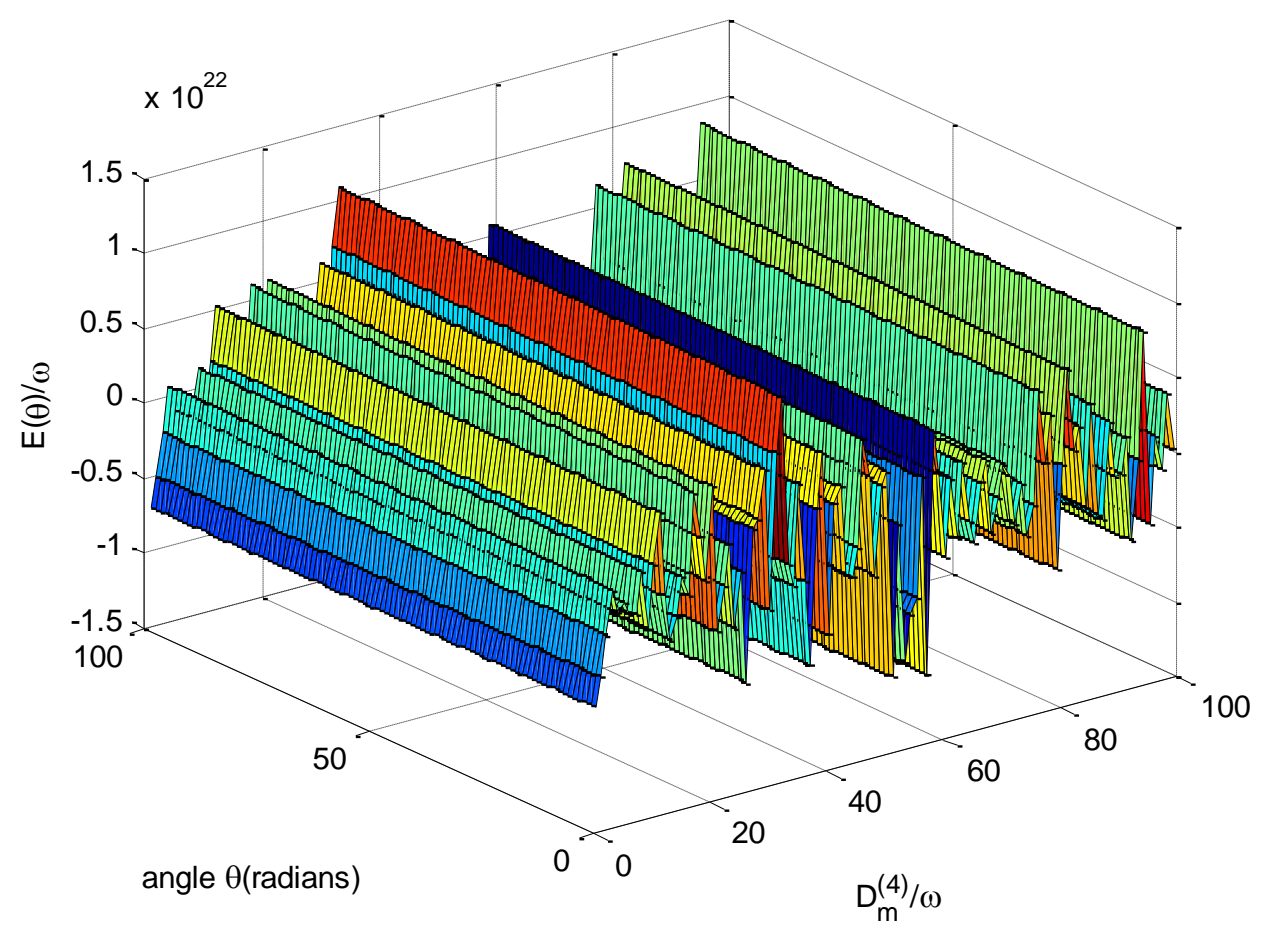

Figure 1: 3-D plot of energy versus angle and fourth order anisotropy 
Then figure 2 shows the 3-D plot of magnetic energy found in equation 11 versus angle and $\frac{D_{m}{ }^{(2)}}{\omega}$ for $\frac{J}{\omega}=\frac{H_{\text {in }}}{\omega}=\frac{H_{\text {out }}}{\omega}=\frac{K_{s}}{\omega}=10$ and $\frac{D_{m}{ }^{(4)}}{\omega}=5$. The energy is a minimum at $\frac{D_{m}{ }^{(2)}}{\omega}=11,19,22,25,59,66$-----etc. This means that the film is easy to magnetize along the easy directions at these values of $\frac{D_{m}{ }^{(2)}}{\omega}=11,19,22,25,59,66$-----etc. In addition, the energy is a maximum at $\frac{D_{m}{ }^{(2)}}{\omega}=5,28,65,81,95,98$-----etc, indicating that the film is difficult to magnetize along the hard directions at these values of $\frac{D_{m}{ }^{(2)}}{\omega}$. According to the graph, there are many easy and hard directions for one particular value of $\frac{D_{m}^{(2)}}{\omega}$.

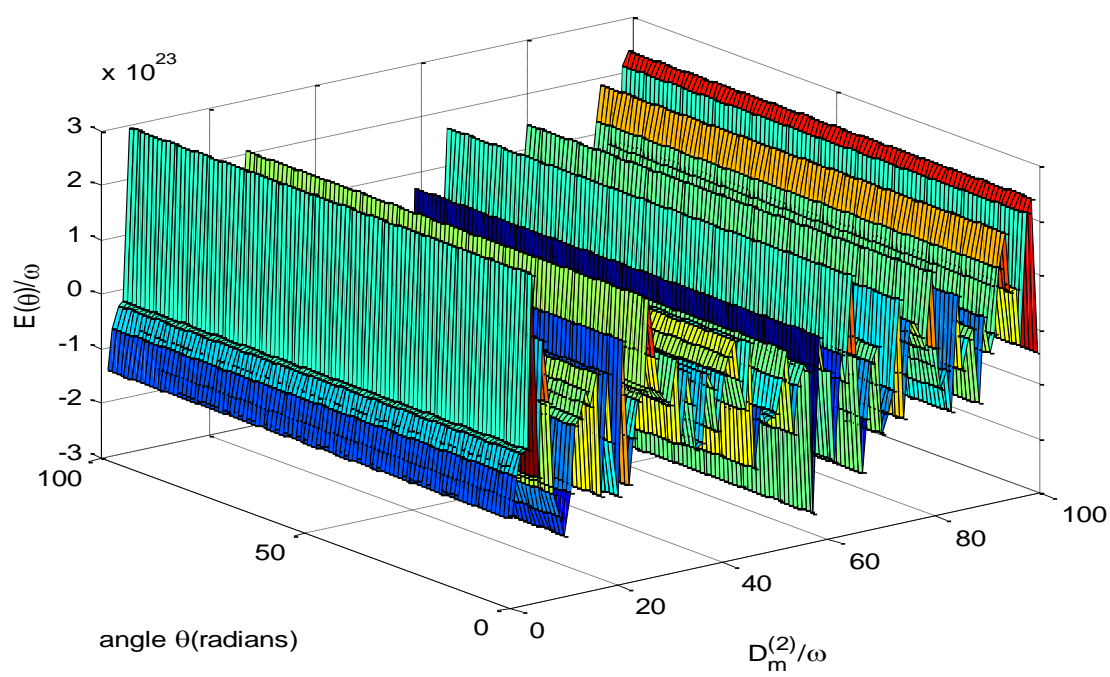

Figure2: 3-D plot of energy versus angle and second order anisotropy

The 2-D plot of magnetic energy versus angle is given in figure 3 for $\frac{J}{\omega}=\frac{D_{m}{ }^{(2)}}{\omega}=\frac{H_{\text {in }}}{\omega}=\frac{H_{\text {out }}}{\omega}=\frac{K_{s}}{\omega}=10$ and $\frac{D_{m}{ }^{(4)}}{\omega}=5$. Here N was taken as 10,000 . One minimum can be observed at $\theta=2.67$ radians $\left(153^{\circ}\right)$ corresponding to the easy direction of magnetization of cobalt ferrite film with 10,000 layers. 


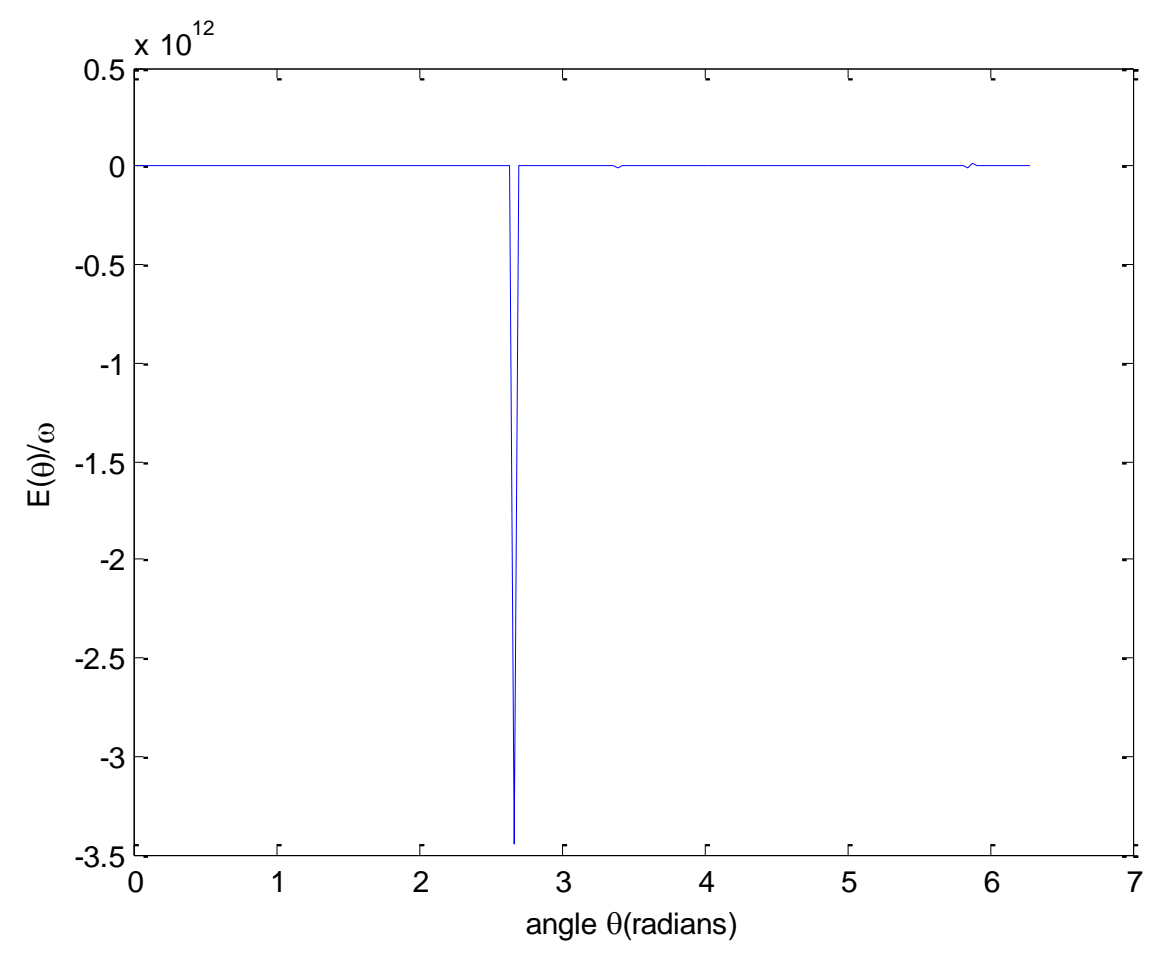

Figure 3: Graph of energy versus azimuthal angle of spin

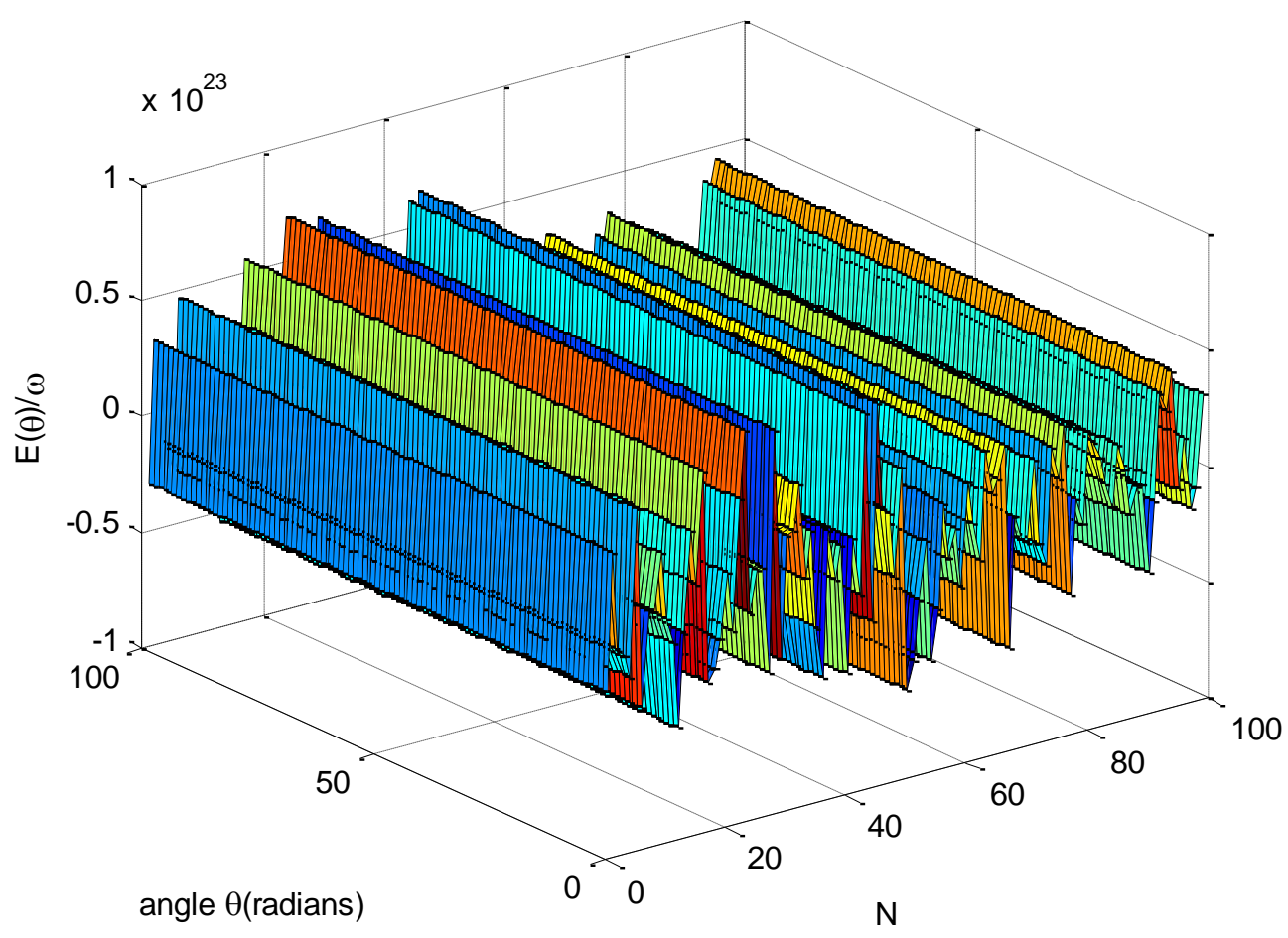

Figure4: 3-D plot of energy versus angle and number of layers of film 
Figure 4 shows the 3-D plot of energy versus angle and number of layers for $\frac{J}{\omega}=\frac{D_{m}{ }^{(2)}}{\omega}=\frac{H_{\text {in }}}{\omega}=\frac{H_{\text {out }}}{\omega}=\frac{K_{s}}{\omega}=10$ and $\frac{D_{m}{ }^{(4)}}{\omega}=5$. The curve has minimum values at $\mathrm{N}=30,52,55,67,88$----etc. This means that the film can be easily align along the easy directions at these $\mathrm{N}$ values. The maximums of the 3 -D plot can be found at $\mathrm{N}=44,46$, $82,95-----e t c$, implying that it is difficult to align the film in magnetically hard direction at these $\mathrm{N}$ values. Some experimental evidences for the dependence of magnetically easy direction on film thickness have been reported for $\mathrm{CrO}_{2}$ (Anwar \& Aarts, 2011).

\section{CONCLUSIONS}

The total magnetic energy found using the third order perturbed Heisenberg Hamiltonian is persuasive that there are minimum and maximum values of energies at certain values of number of layers $(\mathrm{N})$, second order $\left(\frac{D_{m}{ }^{(2)}}{\omega}\right)$ and fourth order magnetic anisotropy $\left(\frac{D_{m}{ }^{(4)}}{\omega}\right)$. This implies that there are magnetically easy and hard directions of the cobalt ferrite film. For $\frac{J}{\omega}=\frac{D_{m}{ }^{(2)}}{\omega}=\frac{H_{\text {in }}}{\omega}=\frac{H_{\text {out }}}{\omega}=\frac{K_{s}}{\omega}=10$, energy is a minimum at $\frac{D_{m}{ }^{(4)}}{\omega}=27,38,41,52,55,58---$ etc and, the energy is a maximum at $\frac{D_{m}{ }^{(4)}}{\omega}=21,33,39,47,---$ etc. For $\quad \frac{J}{\omega}=\frac{H_{\text {in }}}{\omega}=\frac{H_{\text {out }}}{\omega}=\frac{K_{s}}{\omega}=10$ and $\frac{D_{m}{ }^{(4)}}{\omega}=5$, the energy is a minimum at $\frac{D_{m}{ }^{(2)}}{\omega}=11,19,22,25,59,66$-----etc and, the energy is a maximum at $\frac{D_{m}{ }^{(2)}}{\omega}=5,28,65,81,95,98$-----etc. The films can be easily aligned along some certain directions at the above values of $\frac{D_{m}{ }^{(2)}}{\omega}$ and $\frac{D_{m}{ }^{(4)}}{\omega}$ corresponding to energy minimums. 


\section{REFERENCES}

Anwar M.S. and Aarts J., 2011. Inducing supercurrents in thin films of ferromagentic $\mathrm{CrO}_{2}$. Superconductor Science Technology 24: ID 024016.

Araújo C., Almeida B.G., Aguiar M. and Mendes J.A., 2008. Structural and magnetic properties of $\mathrm{CoFe}_{2} \mathrm{O}_{4}$ thin films deposited by laser ablation on $\mathrm{Si}$ (001) substrates. Vacuum 82 (12): 1437-1440.

Barbosa J.G., Pereira M.R., Mendes J.A., Proença M.P., Araújo J.P. and Almeida B.G., 2010. Cobalt ferrite thin films deposited by electrophoresis on p-doped $\mathrm{Si}$ substrates. Journal of Physics: Conference Series 200: ID 072009.

Raghunathan A., Nlebedim I.C., Jiles D.C. and Snyder J.E., 2010. Growth of crystalline Cobalt ferrite thin films at lower temperatures using pulsed laser deposition technique. Journal of Applied Physics 107(9): ID 09A516.

Rajendra S. Gaikwad, Sang-Youn Chae, Rajaram S. Mane, Sung-Hwan Han and OhShim Joo, 2011. Cobalt Ferrite Nanocrystallites for Sustainable Hydrogen Production Application. International Journal of Electrochemistry 2011: ID 729141.

Samarasekara P., 2007. Classical Heisenberg Hamiltonian Solution of Oriented Spinel Ferrimagnetic Thin Films. Electronic Journal of Theoretical Physics 4(15): 187200.

Samarasekara P., Abeyratne M.K. and Dehipawalage S., 2009. Heisenberg Hamiltonian with Second Order Perturbation for Spinel Ferrite Thin Films. Electronic Journal of Theoretical Physics 6(20): 345-356.

Samarasekara P., 2010. Determination of Energy of thick spinel ferrite films using Heisenberg Hamiltonian with second order perturbation. Georgian Electronic scientific Journals: Physics 1(3): 46-52.

Samarasekara P. and Mendoza William A., 2011. Third Order Perturbed Heisenberg Hamiltonian of Spinel Ferrite Ultra-thin films. Georgian Electronic scientific Journals: Physics 1(5): 15-24.

Samarasekara P., 2011. Investigation of Third Order Perturbed Heisenberg Hamiltonian of Thick Spinel Ferrite Films. Inventi Rapid: Algorithm Journal 2(1): 1-3.

Thang P.D., Rijnders G. and Blank D.H.A., 2007. Stress-induced magnetic anisotropy of $\mathrm{CoFe}_{2} \mathrm{O}_{4}$ thin films using pulsed laser deposition. Journal of Magnetism and Magnetic Materials 310 (2): 2621-2623. 